\title{
The effect of dexmedetomidine on status epilepticus in a patient with anti-NMDA receptor encephalitis
}

\author{
Flaminia Coluzzi, MD (D) Albina Angelini, MD • Maurizio Simmaco, MD, \\ PhD - Daniela Alampi, MD · Elisa Alessandri, MD · Eugenio Grassi, RNT • \\ Maria Grazia Monina, MD • Monica Rocco, MD
}

Received: 8 May 2020/Revised: 17 June 2020/Accepted: 17 June 2020/Published online: 15 July 2020

(C) Canadian Anesthesiologists' Society 2020

\section{To the Editor,}

A 35-yr-old Caucasian woman was hospitalized in psychiatry, 8 months after childbirth (G2P2), for psychomotor agitation, visual hallucinations, and incoherent speech. On admission, electroencephalography (EEG), brain imaging (computed tomography and magnetic resonance imaging), and cerebrospinal fluid (CSF) examination showed no abnormalities. On day 7 , she was found unconscious, and she was intubated and

F. Coluzzi, MD (ه)

Department of Medical and Surgical Sciences and Biotechnologies, Sapienza University of Rome, Latina, Italy

e-mail: flaminia.coluzzi@uniroma1.it

Unit of Anesthesia and Intensive Care Medicine, Sant'Andrea University Hospital, Rome, Italy

A. Angelini, MDE. Alessandri, MDM. G. Monina, MD Unit of Anesthesia and Intensive Care Medicine, Sant'Andrea University Hospital, Rome, Italy

M. Simmaco, MD, PhD

Department of Neurosciences, Mental Health and Sensory

Organs, Sapienza University of Rome, Rome, Italy

Advanced Molecular Diagnostic Unit, Sant'Andrea University Hospital, Rome, Italy

D. Alampi, MDM. Rocco, MD

Unit of Anesthesia and Intensive Care Medicine, Sant'Andrea University Hospital, Rome, Italy

Department of Clinical and Surgical Translational Medicine, Sapienza University of Rome, Rome, Italy

E. Grassi, RNT

Unit of Neurophysiopathology, University Hospital

Sant'Andrea, Rome, Italy admitted to the intensive care unit (ICU). After successful liberation from mechanical ventilation, she developed status epilepticus (SE) alternating with periods of agitation. SE persisted despite combined treatment with phenytoin sodium, levetiracetam, and delorazepam. Intravenous dexmedetomidine $\left(0.4 \mu \mathrm{g} \cdot \mathrm{kg}^{-1} \cdot \mathrm{hr}^{-1}\right)$ was administrated for conscious sedation, according to local protocols, and improvement from the SE was observed in 30 min, as documented with EEG tracings. Electroencephalography spectral analysis showed a significant increase in beta activity, which is typical of dexmedetomidine (Figure). SE relapsed after dexmedetomidine interruption and resolved when the infusion was restarted. Oral lacosamide was prescribed as second-line therapy for drug-resistant SE, but the patient's clinical condition worsened and dysautonomic signs became more evident, including hypertension, tachycardia, and a very high body temperature $\left(>39^{\circ} \mathrm{C}\right)$. Lumbar puncture was repeated to investigate an autoimmune etiology and anti-N-methyl-D-aspartate receptor (NMDA-R) antibodies were detected in the CSF. Immunoglobulins, high-dose corticosteroids, and subsequent plasmapheresis were administered with success.

$\mathrm{N}$-methyl-D-aspartate receptor antibodies are a rare cause of autoimmune encephalitis, often misdiagnosed with psychosis, which typically leads to seizures and drugresistant SE. This is an unusual case, describing the anticonvulsant effect of dexmedetomidine in SE due to NMDA-R encephalitis.

Contradictory results have been reported on the effects of the alpha-2 agonist dexmedetomidine in epilepsy. In animals, dexmedetomidine has been shown both to reduce and increase the seizure threshold in local anestheticinduced epilepsy. In rats, the anti-epileptic effect of 
(a) Before dexmedetomidine
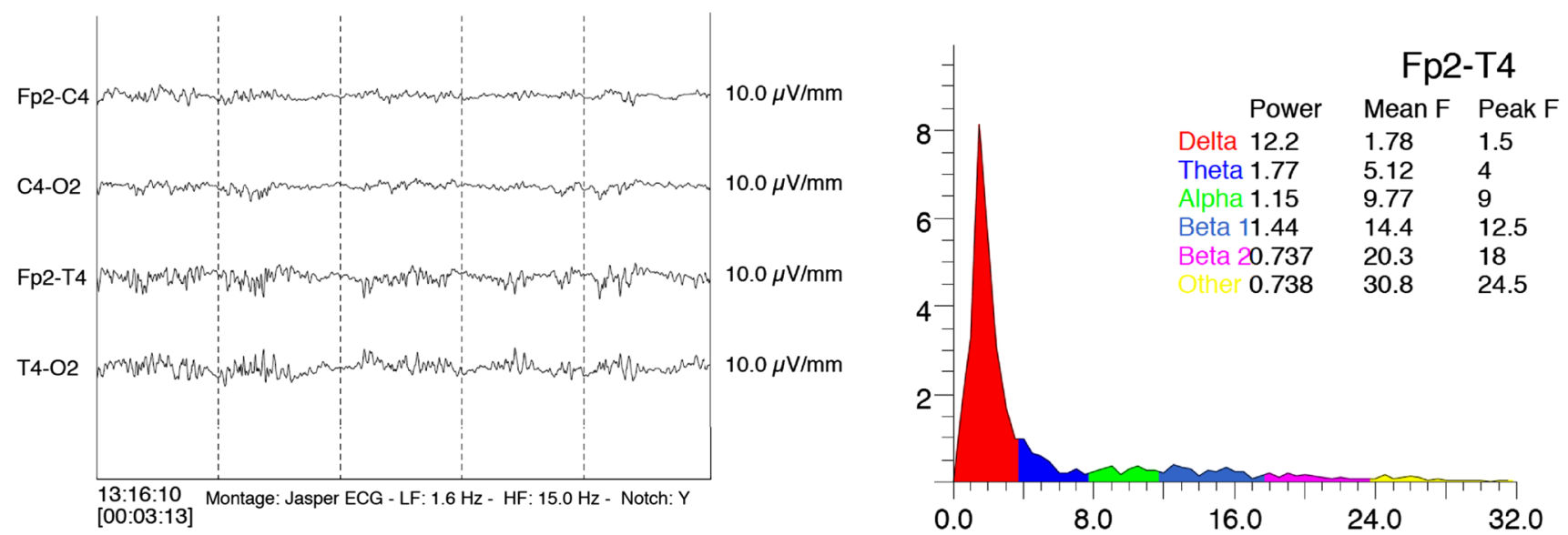

(b) After dexmedetomidine
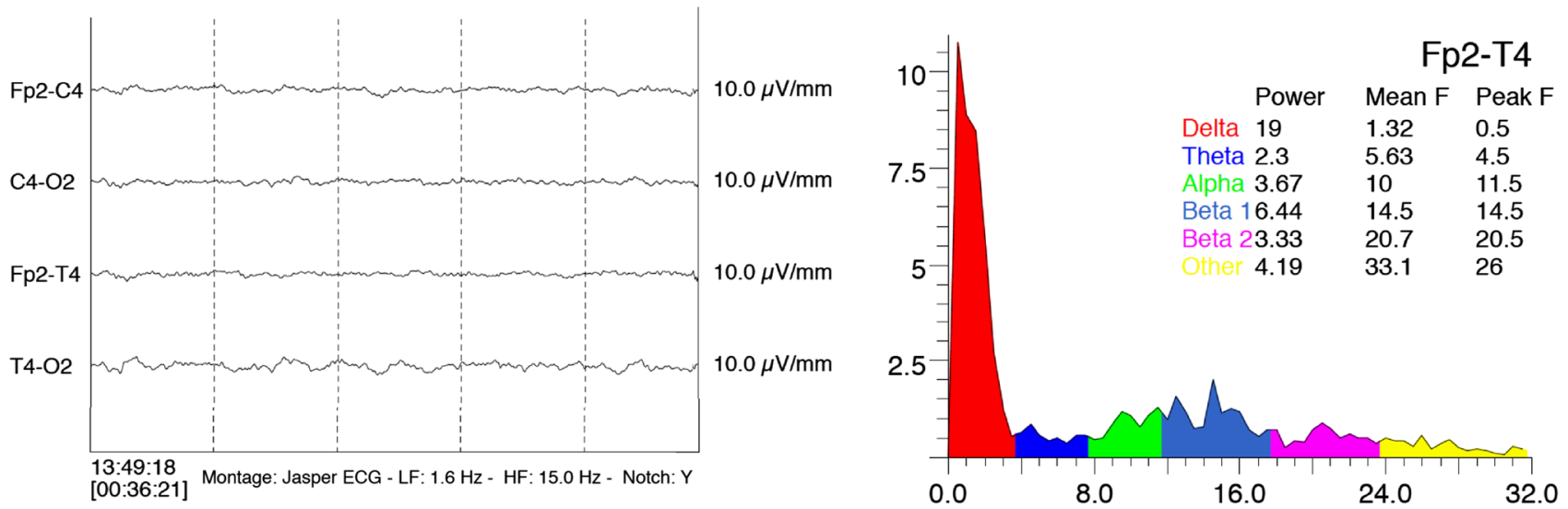

Figure Electroencephalography (EEG) tracings and spectral analysis. a) Electroencephalography tracing before dexmedetomidine showed muscular artifacts (myogenic potentials generated by masticatory activity) with specific temporal elements (spikes), followed by reduction of amplitude of cortical bioelectric activity. Electroencephalography spectral analysis showed diffuse delta activity with associated theta activity. b) Thirty minutes after

dexmedetomidine was reversed by the alpha-2 adrenoreceptor antagonist atipamezole, further suggesting a role for these receptors in seizures. ${ }^{1}$ In humans, dexmedetomidine-induced seizures have been described in both neonates and adults. Nevertheless, a case report recently showed that dexmedetomidine infusion at 0.6 $\mu \mathrm{g} \cdot \mathrm{kg}^{-1} \cdot \mathrm{hr}^{-1}$ successfully treated drug-resistant SE. ${ }^{2}$

Alpha- 2 adrenoreceptors are located on both sides of the synaptic cleft. Therefore, alpha- 2 agonist drugs may have opposite effects on noradrenergic transmission. At the presynaptic level, dexmedetomidine decreases noradrenaline release and could have proconvulsant activity. Conversely, at the postsynaptic level, dexmedetomidine, by increasing noradrenaline transmission, may exert anticonvulsant action. Therefore, dexmedetomidine infusion $\left(0.4 \mu \mathrm{g} \cdot \mathrm{kg}^{-1} \cdot \mathrm{hr}^{-}\right)$, EEG tracing showed low-amplitude, high-frequency activity and rapid potentials of probable pharmacological origin. On EEG spectral analysis, an increase in beta activity was observed, mainly in beta1, which lasted through the EEG registration. Both traces are displayed with a sensitivity of $10 \mathrm{mV} / \mathrm{mm}$, high frequency filter $=15.0 \mathrm{~Hz}$, and low frequency filter $=1.6 \mathrm{~Hz}$.

as the balance between these two activities may be affected by different factors, the net effect of dexmedetomidine in epilepsy is not completely clear. ${ }^{3}$

Another intriguing question is the exact relationship between dexmedetomidine and NMDA-R. N-methyl-Daspartate receptors are ionotropic glutamate receptors that play a key role in excitatory synaptic transmission. Dexmedetomidine can depress NMDA-R-mediated transmission. $\mathrm{N}$-methyl-D-aspartate receptors are involved in opioid-induced hyperalgesia, and dexmedetomidine has been shown to prevent remifentanil-induced hyperalgesia. Dexmedetomidine exerts its activity on G-protein coupled alpha-2 adrenoreceptors through inhibition of cyclic adenosine monophosphate and reduction of phosphokinase A (PKA). 
As PKA can potentiate NMDA-R activity, it is reasonable to assume that dexmedetomidine could reduce the phosphorylation of the NR2B subunit, subsequently alterating NMDA-R trafficking. ${ }^{4}$ NMDA-R antagonists, such as ketamine, are considered a good approach for the treatment of SE; therefore, we could hypothesize that, in our patient, dexmedetomidine suppressed the spike activity, by reducing phosphorylation, and indirectly targeting the NMDA-R. Dexmedetomidine has been previously used in the postoperative setting of a woman affected by NMDA-R encephalitis, to achieve an adequate level of sedation. ${ }^{5}$

Our observations suggest a possible role for dexmedetomidine in the management of drug-resistant SE in the ICU. In patients with NMDA-R encephalitis and seizures, dexmedetomidine could be a useful bridge between typical pharmacological approaches and plasmapheresis.

Disclosures None.

\section{Funding statement None.}

Editorial responsibility This submission was handled by Dr. Sangeeta Mehta, Associate Editor, Canadian Journal of Anesthesia.

\section{References}

1. McCarren HS, Arbutus JA, Ardinger C, Dunn EN, Jackson CE, McDonough JH. Dexmedetomidine stops benzodiazepinerefractory nerve agent-induced status epilepticus. Epilepsy Res 2018; DOI: https://doi.org/10.1016/j.eplepsyres.2018.01.010.

2. Obara S, Kakinouchi K, Honda J, Noji Y, Hanayama C, Murakawa $M$. Dexmedetomidine administration in a patient with status epilepticus under color density spectral array monitoring. JA Clin Rep 2019; DOI: https://doi.org/10.1186/s40981-019-0234-1.

3. Fitzgerald PJ. Is elevated norepinephrine an etiological factor in some cases of epilepsy? Seizure 2010; 19: 311-8.

4. Yuan $Y$, Sun Z, Chen $Y$, et al. Prevention of remifentanil induced postoperative hyperalgesia by dexmedetomidine via regulating the trafficking and function of spinal NMDA receptors as well as PKC and CaMKII level in vivo and in vitro. PLoS One 2017; DOI: https://doi.org/10.1371/journal.pone.0171348.

5. Yamanaka D, Kawano T, Tateiwa H, Iwata H, Locatelli FM, Yokoyama $M$. Successful management of dexmedetomidine for postoperative intensive care sedation in a patient with anti-NMDA receptor encephalitis: a case report and animal experiment. Springerplus 2016; DOI: https://doi.org/10.1186/s40064-0163079-3.

Publisher's Note Springer Nature remains neutral with regard to jurisdictional claims in published maps and institutional affiliations. 